\title{
FOLDNA, a Web Server for Self-Assembled DNA Nanostructure Autoscaffolds and Autostaples
}

\author{
Chensheng Zhou, Heng Luo, Xiaolu Feng, Xingwang Li, Jie Zhu, Lin He, and Can Li
}

Bio-X Institutes, Shanghai Jiao Tong University, 1954 Huashan Road, Shanghai 200240, China

Correspondence should be addressed to Can Li, lican@sjtu.edu.cn

Received 3 April 2012; Revised 23 July 2012; Accepted 10 August 2012

Academic Editor: Menka Jain

Copyright ( $) 2012$ Chensheng Zhou et al. This is an open access article distributed under the Creative Commons Attribution License, which permits unrestricted use, distribution, and reproduction in any medium, provided the original work is properly cited.

DNA self-assembly is a nanotechnology that folds DNA into desired shapes. Self-assembled DNA nanostructures, also known as origami, are increasingly valuable in nanomaterial and biosensing applications. Two ways to use DNA nanostructures in medicine are to form nanoarrays, and to work as vehicles in drug delivery. The DNA nanostructures perform well as a biomaterial in these areas because they have spatially addressable and size controllable properties. However, manually designing complementary DNA sequences for self-assembly is a technically demanding and time consuming task, which makes it advantageous for computers to do this job instead. We have developed a web server, FOLDNA, which can automatically design 2D self-assembled DNA nanostructures according to custom pictures and scaffold sequences provided by the users. It is the first web server to provide an entirely automatic design of self-assembled DNA nanostructure, and it takes merely a second to generate comprehensive information for molecular experiments including: scaffold DNA pathways, staple DNA directions, and staple DNA sequences. This program could save as much as several hours in the designing step for each DNA nanostructure. We randomly selected some shapes and corresponding outputs from our server and validated its performance in molecular experiments.

\section{Introduction}

When Watson and Crick discovered the nucleic acid pairing rules in 1953, the theoretical foundation of self-assembled DNA was established. In 1982, Seeman laid the foundation of "structural DNA Nanotechnology" by folding DNA into lattices [1]. Later in 2006, Rothemund initiated spatially restricted 2D self-assembled DNA structure by using a bottom-up method, which is also known as DNA origami $[2,3]$. In the same year, asymmetric self-assembled DNA nanostructure was folded by Qian et al. [4]. Since then, applications of DNA nanostructures in research and diagnosis have been continuously developed, such as methods to examine single nucleotide polymorphism on flat DNA origami, to attach antibodies onto tiles and to perform RNA hybridization assays [5-8].

Self-assembled DNA is built by a long single strand scaffold DNA and a lot of short single strand DNA known as staples. The number of staples is decided by the length of the scaffold DNA, which is typically 7 kilobase-long.
Using bottom-up fabrication methods, the scaffold DNA is folded to form the shape of the nanostructure, while the staple DNA fix the scaffold DNA, thus forming the twodimensional DNA nanostructure. With the development of DNA nanotechnology, software for computer-aided design of nucleic acids was developed to help with the calculation of DNA nanostructure [9-15]. By using the software, the efficiency of designing self-assembled DNA nanostructure has been dramatically increased. However, there are still several manual steps for designing the nanostructure, including modification of border staples and calculation scaffold DNA length in each line, which require a full understanding of the DNA origami technical details. Hence an automatic design tool which provides a more easy access to apply the DNA nanofabrication tools to researchers in related areas, for instance, nanoparticle base drug delivery, will introduce selfassembled DNA technology to more interdisciplinary areas [16].

In our server FOLDNA, the pathway of the scaffold is automatically generated according to the shape of a 


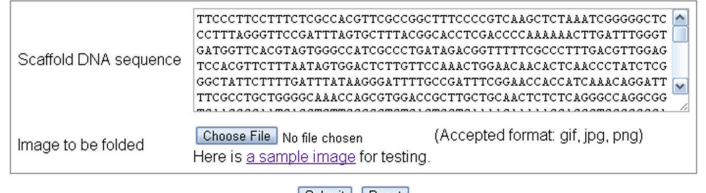

Submit Reset

Figure 1: Screenshot from FOLDNA home page. In the web server FOLDNA, users can upload a monochrome image and a scaffold DNA sequence. Then the server will automatically design the whole nanostructure. Here we have a default scaffold DNA sequence (M13mp18) provided. It can be customized to any desired sequence.

given picture. Then the staple DNA strands, typically 32 nucleotides (nt) each, are generated to pair with the scaffold. We fill in the staple DNA according to the DNA helix torque angle, ensuring the final nanostructure is flat according to Rothemund's method [2]. With the help of FOLDNA, the entire process of DNA nanostructure design is simplified to image preparation, checking the results, and ordering oligonucleotides to carry out experiments. This open server is available at http://www.bio-x.cn/foldna/.

\section{Results}

2.1. Server Results. The web server FOLDNA offers a userfriendly interface (Figure 1). An example scaffold DNA (M13mp18), which can be customized by the user, is provided for a quick test. By submitting a desired shape of DNA nanostructure as a monochrome picture, along with the scaffold DNA sequence, users can obtain complete sequences of staple DNA which can hybridize onto the scaffold DNA to form the desired shape. No matter what the size the given picture is, the program will automatically adjust the picture so that the long scaffold DNA can be optimally filled into it. The results displayed in FOLDNA contain the pathway and sequence of the folded scaffold DNA and staple DNA, which can be directly used in the experiments. Finally, wet-lab experiments are conducted to validate the results from the program (Figure 2).

\section{Interpretation of Results Displayed in Web Server}

3.1. Digitized Image. A digitized image is displayed together with the uploaded image. The digitized image outline indicates how the image is filled by the scaffold DNA. Each number in the digitized image stands for one basic building brick (brick for abbreviation). Here, the width of each brick is equal to 8 bases of nucleic acid, while the height is equal to 2 rows. When rectangular bricks are filled into the monochrome image, bricks around the border lines will be either picked or abandoned. We try to make the best use of the scaffold sequence and avoid left single strand to form mismatches. Here, we use a floating point value system to evaluate the percentage of the black-colored area and determine whether or not a border brick should be picked.
This percentage cannot be set as a fixed value because a rigid value does not work well for all images. Therefore, a floating point is calculated according to each image, under the precondition that there are enough scaffold DNA to fill them, thereby ensuring the maximum number of border bricks are included. As a result, the left length of the scaffold DNA in the web page is normally no more than 50 bases.

3.2. Scaffold Structure. In our program, the default long single stand DNA that acts as scaffold is M13mp18, a $7 \mathrm{~kb}$ single strand DNA from bacteriophage M13. However, if a different scaffold is desired, users can customize their own sequence. Depending on the length of the uploaded sequence, the program zooms the image in or out to fit the uploaded template before generating the scaffold pathway. FOLDNA is able to process complicated images such as images with holes, or images with erratic borders.

To maximize the function of our web server, we have worked out a program that can deal with any continual image. Our program accepts shapes with holes or indentations at any location. The bottom-up designing method is specially written to ensure all complicated shapes can be reasonably filled up by the scaffold sequences.

3.3. Staple Structure. Since scaffold DNA mainly goes crabwise, it needs something vertical to fix it. Staple DNA exactly binds the neighboring scaffold strands. According to Rothemund's paper [2], staples were first designed to bind neighboring upper and lower scaffold DNA, with about 8 bases on the upper one and another 8 bases on the lower one. Then, to make it more stable, adjacent staples are linked, which increases the average staple length to about 32 bases. On the top and bottom edges, staples are prolonged or cut by the program to ensure the edge is completely paired. The shortest staple DNA generated by the server is 24 bases, while the longest staple is 40 bases. This range ensures a reasonable melting temperature and a reasonable length for synthesis and purification.

With user determined scaffold DNA length, sequence, and desired shape, the staple DNA sequences are calculated automatically.

3.4. Staple Sequences. On the web server, the staple DNA sequence can be downloaded as an isolated file. When closing the webpage, the digitized image will expire, but the link to the staple sequence will still be valid until the server space has been filled, at which point old files will be replaced.

\section{Experimental Results}

Designing results from FOLDNA are validated by molecular experiments. They are tested by uploading a simple image of a hexagon and complex images with concavity and holes, along with the example scaffold DNA (M13mp18), to examine the capability of our server. We ordered staple DNA according to its output and performed the selfassembly experiments of hybridizing the staple DNA onto the scaffold DNA. The shapes of assembled DNA nanostructures 


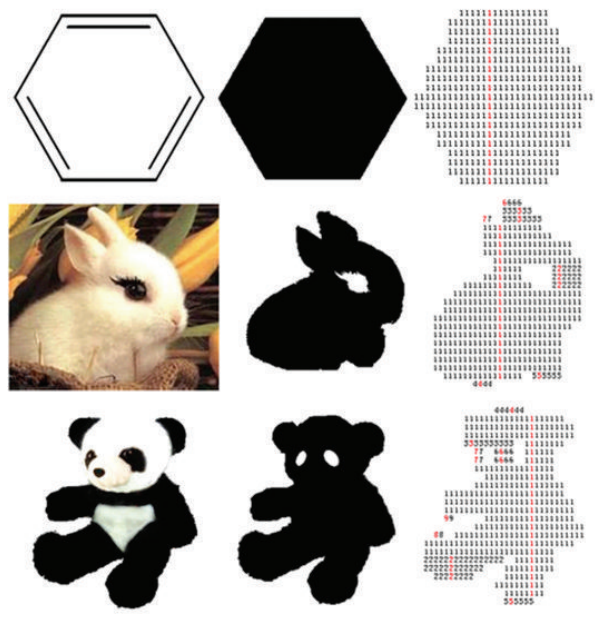

(a)

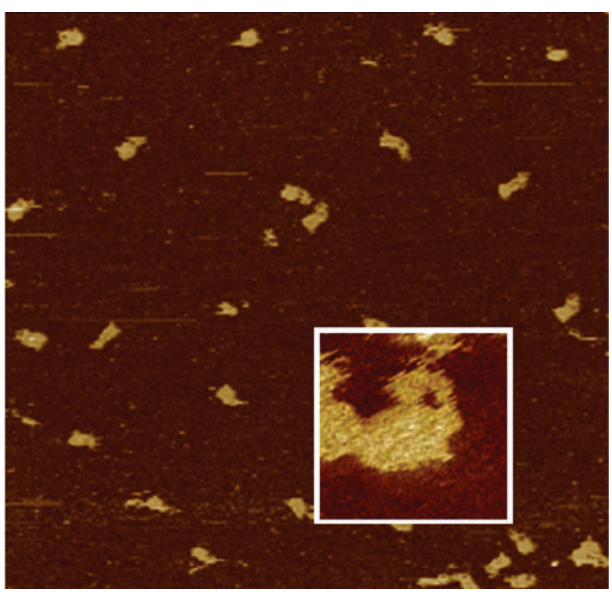

(c)

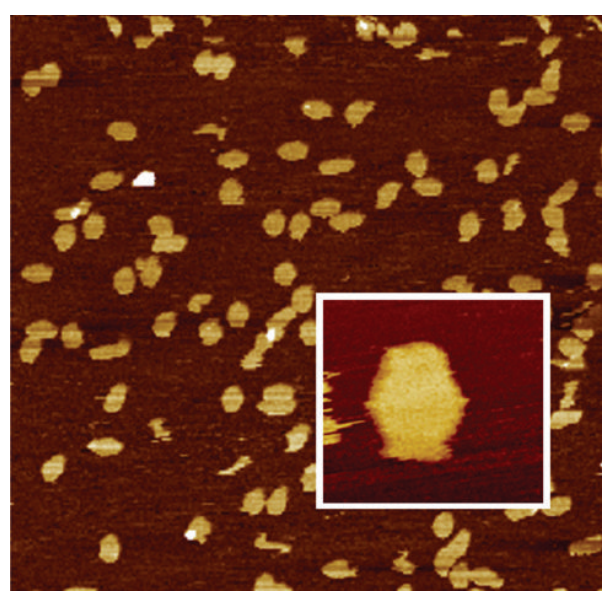

(b)

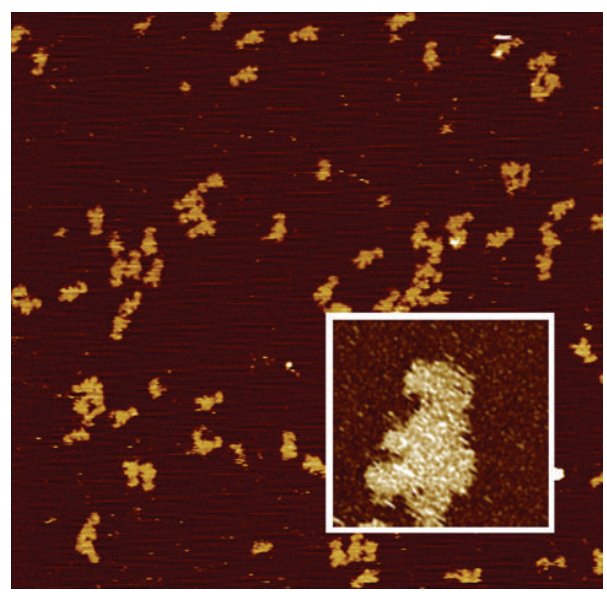

(d)

FIgURE 2: Examples for DNA nanostructure design. (a) left column: object picture; middle column: monochrome image, user uploaded; right column: digitized image, according to the user uploaded image. (b, c, d), wet-lab products observed by AFM. Uploaded image and digitized image are screenshots from our web server. In the digitized images, scaffold ssDNA forms seams on the border and along the red lines. The selected shapes for validation are a hexagon, a rabbit and a panda (a). The corresponding results are (b), (c) and (d). The pathways for the scaffold and staple DNA as well as the sequence lists for staple DNA we used for wet-lab experiments are shown in the Supplementary Materials (see Supplementary Materials available online at doi:10.1155/2012/453953).

are observed through AFM nanoscopes. We found the nanostructures were well folded according to the desired shapes, validating the power of applying our server in automatic designing of 2D DNA nanostructures. As the broad applications of 2D DNA nanostructures, such as examining single nucleotide polymorphism on DNA origami flat, attaching antibodies onto tiles and RNA hybridization assays, are continuously discovered, FOLDNA will be an efficient and powerful tool in designing either complicated or high through-put DNA nanostructures for experiments and further research.

\section{Discussion}

Since computer aided design of self-assembled DNA nanostructures was developed, software has provided significant convenience. Such software offers automatic design of staple sequences according to customer scaffold structure. A manual design of a scaffold pathway is tedious and troublesome, and is not yet included in such computer applications. Thus, a smart program which works out all steps above, and can carry self-assembled DNA nanostructures into execution without using other calculation platforms will increase the accessibility and the user base of self-assembled DNA nanostructures.

Web server FOLDNA is such a program. It is the first program to provide entirely automatic design of scaffold DNA pathway according to any given continuous image. With the same customer uploaded image, a longer scaffold DNA would provide a larger structure and better details if the design is complex. Obviously, a longer scaffold needs more work in calculation. Thus, the users can customize their own nanostructures with any kind of scaffold DNA for potential uses such as probable binding, recognition, 


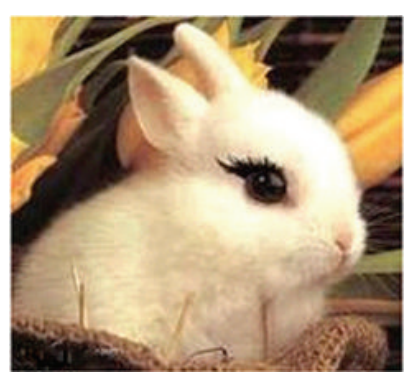

(a)

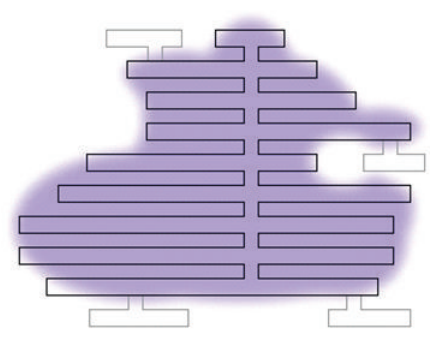

(b)

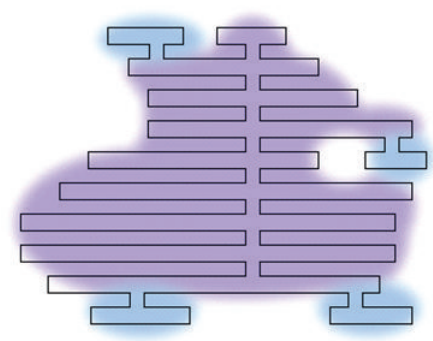

(c)

FIGURE 3: Example of scaffold prolong step by step. (a), the desired shape that need to be filled by scaffold. (b) major part along the first seam is filled up. (c) the second part along the second seam is derived from one point of the first part. Then, minor parts are filled by strands comes from its neighboring part, until the whole picture is filled.
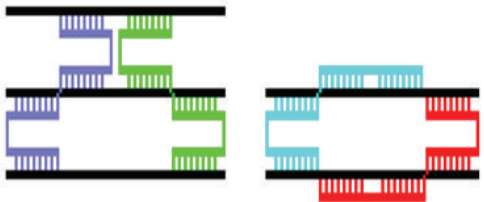

(a)

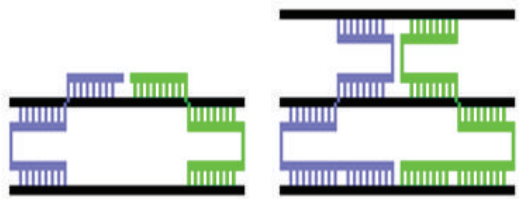

(b)

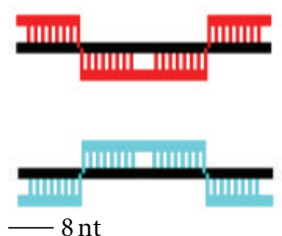

(c)

FIgure 4: Staple DNA types. (a) general and special type staples, left: Z staple (violet) and S staple (green); right: L staple (blue) and R staple (red); (b and c), variations on the staples when located on the top and bottom edges: (b) S (violet) and Z (green) type staple variations when meeting the top of a seam (left) and the bottom of a seam (right); (c) L (red) and R (blue) type staple variations on the top edge (top) and the bottom edge (bottom).

affinity, or detection of special chemicals or macromolecules. The traditional designing method of DNA nanostructures requires you to first draw the monochrome picture onto a lattice and calculate the total area of the image by counting units. Then, the pathway that the scaffold DNA can cover is inscribed onto the lattice, and the approximate length of DNA in each line and the total number of lines in all are calculated manually. Finally the nanostructure is separated into several parts and designed part by part, ensuring the scaffold DNA to be continuous at any point. However, with the help of our FOLDNA, all steps above can be executed instantly with accuracy and quality. FOLDNA can create a planned portrayal, no matter how complicated the desired image is, or whether the image has holes or caves inside or at its edges.

\section{Materials and Methods}

6.1. Oligonucleotides. Single strand DNA M13mp18 was purchased from New England Biolabs, stored at $10 \mathrm{mM}$ Tris$\mathrm{HCl}$ ( $\mathrm{pH}$ 7.5), 1mM EDTA. Staples DNA are ordered from GeneRay, purified by PAGE.

6.2. Methods of Forming DNA Nanostructures. In the reaction, staples are diluted to approximately $0.025 \mathrm{uM}$. Each sample (30 ul) contains $0.5 \mathrm{ul}$ scaffold DNA M13 mp18, 7.5 ul TAE-Mg2+ buffer and 22 ul diluted staple DNA. Selfassembled DNA is performed in PCR machine. The mixture of oligonucleotides is denatured at 95 degrees for 2 minutes, then slowly cooling down to 20 degrees in approximately 2 hours, at the speed of 0.1 degrees every 10 seconds, for hybridization. After the assembly step is finished, the samples are stored at 4 degrees till loading for AFM imaging.

6.3. AFM Imaging. Atomic force microscope imaging was conducted in Veeco multimode nanoscope III, tapping mode in liquid phase. E scanner with NP/S tip is used for imaging. 2 ul sample was loaded on fresh cleaved mica, and additional 20 ul TAE-Mg2+ buffer were added into cantilever before place the holder into the box. The tapping amplitude setpoint was about $0.4 \mathrm{mV}$, using approximately $9.7 \mathrm{kHz}$ resonance drive frequency.

6.4. Server-Side Calculation of Autoscaffold. When a monochrome image of desired shape is uploaded, it is resized to fit the length of the scaffold. The server makes the best use of the scaffold and keeps both ends of the scaffold DNA close enough to each other so that the program is compatible with either circular or linear DNA [17]. The monochrome image is then filled up by bricks as basic units which are shown as numbers in the digitized image of the output. Each brick is built up by 2 parallel lines of $8 \mathrm{nt}$ and the number of the bricks in each line is even, ensuring $16 \mathrm{nt}$ as the basic unit for the scaffold.

Next, in order to ensure the entire structure is flat after assembly, the server calculates the seams and pathway for the scaffold DNA according to the methods used by previous publications $[2,15]$. By taking the advantage of 
these methods, the seams will go across all the horizontal lines of the monochrome image, making sure that the entire image is covered by the scaffold DNA (Figure 3). The top of the longest seam is set as the default start point of the scaffold to be filled in with a default direction towards the right.

6.5. Server-Side Calculation of Autostaple. Autostaple has been already used in some software [10, 13, 15], however, with the involvement of extra short staples on the edges, manual modification of the staple DNA is necessary. However, with the help of our server, entirely complementary staples are automatically generated and filled into the bricks according to the scaffold pathway. In our program, staples are mainly divided into 2 types, with 2 subtypes in each. The general type includes $S$ and $Z$ subtypes, while the special type includes L and R subtypes (Figure 4).

$\mathrm{S}$ and $\mathrm{Z}$ subtypes are a general choice for complementation of the scaffold. However, this rule cannot work when a seam is located at even bricks away from the edge. The nanostructure will separate apart along the seam if this general type of staples is used. At this point, L and R subtypes are applied as to staple together the scaffold DNA at both sides of the seams.

On the top and bottom edges, the staples have a variation. On the top, the $S$ and $Z$ subtypes of staples have no space to extend on the top so they are 8 bases less than their general type. Meanwhile, on the bottom, they are extended 8 bases longer to complement the scaffold. For the L and R subtypes of staples, there is also a variation on the top and bottomthese staples are prolonged into a straight line.

\section{Authors' Contribution}

C. Zhou and H. Luo contributed equally to this work.

\section{Acknowledgments}

The authors thank the National Key Technology R\&D Program (2012BAI01B09), the 973 Program (2010CB529600), and the National Nature Science Foundation of China (81121001) for the financial support.

\section{References}

[1] N. C. Seeman, "Nucleic acid junctions and lattices," Journal of Theoretical Biology, vol. 99, no. 2, pp. 237-247, 1982.

[2] P. W. K. Rothemund, "Folding DNA to create nanoscale shapes and patterns," Nature, vol. 440, no. 7082, pp. 297-302, 2006.

[3] R. Chhabra, J. Sharma, Y. Liu, S. Rinker, and H. Yan, "DNA self-assembly for nanomedicine," Advanced Drug Delivery Reviews, vol. 62, no. 6, pp. 617-625, 2010.

[4] L. Qian, Y. Wang, Z. Zhang et al., "Analogic China map constructed by DNA," Chinese Science Bulletin, vol. 51, no. 24, pp. 2973-2976, 2006.

[5] Y. He, Y. Tian, A. E. Ribbe, and C. Mao, "Antibody nanoarrays with a pitch of $\sim 20$ nanometers," Journal of the American Chemical Society, vol. 128, no. 39, pp. 12664-12665, 2006.

[6] Y. Ke, S. Lindsay, Y. Chang, Y. Liu, and H. Yan, "Self-assembled water-soluble nucleic acid probe tiles for label-free RNA hybridization assays," Science, vol. 319, no. 5860, pp. 180-183, 2008.

[7] Z. Zhang, Y. Wang, C. Fan et al., "Asymmetric DNA origami for spatially addressable and index-free solution-phase DNA chips," Advanced Materials, vol. 22, no. 24, pp. 2672-2675, 2010.

[8] Z. Zhang, D. Zeng, H. Ma et al., "A DNA-origami chip platform for label-free SNP genotyping using toehold-mediated strand displacement," Small, vol. 6, no. 17, pp. 1854-1858, 2010 .

[9] S. M. Douglas, H. Dietz, T. Liedl, B. Högberg, F. Graf, and W. M. Shih, "Self-assembly of DNA into nanoscale threedimensional shapes," Nature, vol. 459, no. 7245, pp. 414-418, 2009.

[10] S. M. Douglas, A. H. Marblestone, S. Teerapittayanon, A. Vazquez, G. M. Church, and W. M. Shih, "Rapid prototyping of 3D DNA-origami shapes with caDNAno," Nucleic Acids Research, vol. 37, no. 15, pp. 5001-5006, 2009.

[11] “NanoEngineer-1," Nanorex Inc., Bloomfield Hills, Mich, USA, http://www.nanorex.com.

[12] J. Zhu, B. Wei, Y. Yuan, and Y. Mi, "UNIQUIMER 3D, a software system for structural DNA nanotechnology design, analysis and evaluation," Nucleic Acids Research, vol. 37, no. 7, pp. 2164-2175, 2009.

[13] C. S. Andersen, H. Yan, and K. V. Gothelf, "Bridging one helical turn in double-stranded DNA by templated dimerization of molecular rods," Angewandte Chemie, vol. 47, no. 30, pp. 5569-5572, 2008.

[14] J. J. Birac, W. B. Sherman, J. Kopatsch, P. E. Constantinou, and N. C. Seeman, "Architecture with GIDEON, a program for design in structural DNA nanotechnology," Journal of Molecular Graphics and Modelling, vol. 25, no. 4, pp. 470-480, 2006.

[15] E. S. Andersen, M. Dong, M. M. Nielsen et al., "DNA origami design of dolphin-shaped structures with flexible tails," ACS Nano, vol. 2, no. 6, pp. 1213-1218, 2008.

[16] J. Li, H. Pei, B. Zhu et al., "Self-assembled multivalent DNA nanostructures for noninvasive intracellular delivery of immunostimulatory CpG oligonucleotides," ACS Nano, vol. 5, no. 11, pp. 8783-8789, 2011.

[17] E. S. Andersen, M. Dong, M. M. Nielsen et al., "Self-assembly of a nanoscale DNA box with a controllable lid," Nature, vol. 459, no. 7243, pp. 73-76, 2009. 

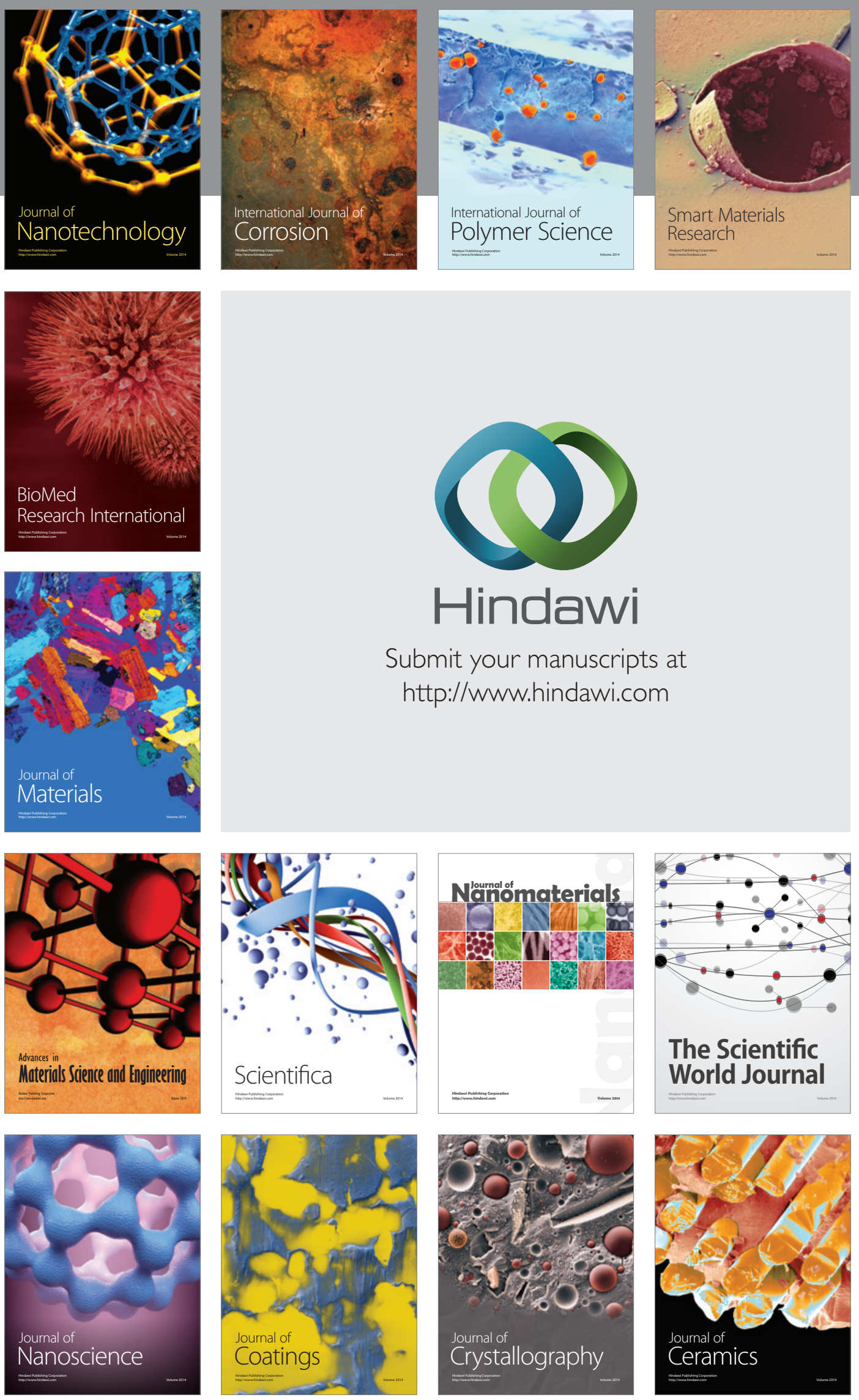

The Scientific World Journal

Submit your manuscripts at

http://www.hindawi.com

\section{World Journal}

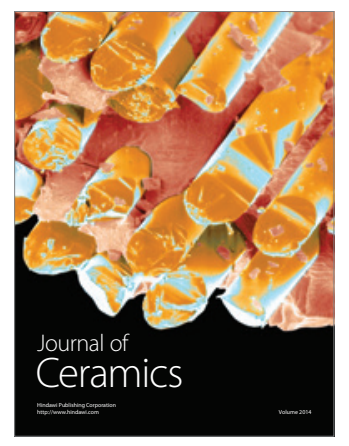

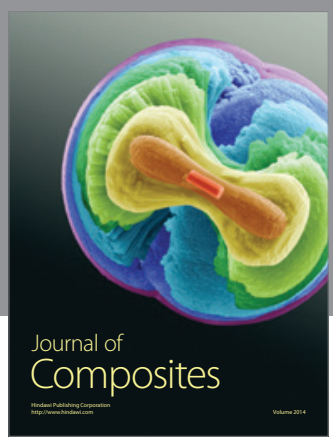
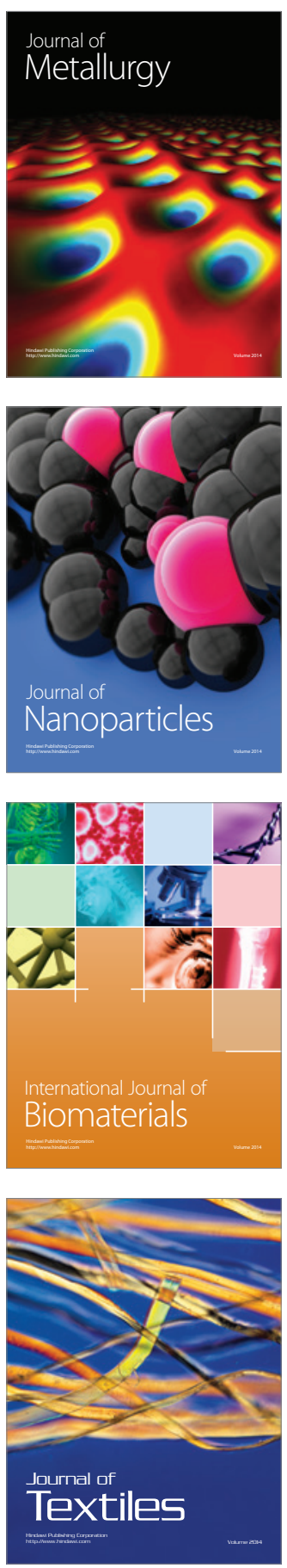